\title{
Estrogen receptor $\beta$ agonist enhances temozolomide sensitivity of glioma cells by inhibiting PI3K/AKT/mTOR pathway
}

\author{
XIAOYANG LIU ${ }^{1,2}$, LIBO WANG ${ }^{2}$, JIAJUN CHEN ${ }^{2}$, QI LING $^{3}$, HONGFEI WANG ${ }^{4}$, \\ SHILIN LI ${ }^{5}$, LIMING LI ${ }^{5}$, SHUPING YANG ${ }^{5}$, MINGYING XIA ${ }^{5}$ and LING JING ${ }^{1}$ \\ ${ }^{1}$ School of Pharmaceutical Sciences, Jilin University, Changchun, Jilin 130021; ${ }^{2}$ China-Japan Union Hospital, Jilin University, \\ Changchun, Jilin 130033; ${ }^{3}$ Jilin Medical College, Jilin, Jilin 132013; ${ }^{4}$ Department of Vascular Surgery, Changhai Hospital, \\ Second Military Medical University, Shanghai 200433; ${ }^{5}$ MOE Key Laboratory of Contemporary Anthropology and \\ State Key Laboratory of Genetic Engineering and School of Life Sciences, Fudan University, \\ Shanghai 200433, P.R. China
}

Received March 20, 2014; Accepted September 19, 2014

DOI: $10.3892 / \mathrm{mmr} .2014 .2811$

\begin{abstract}
Glioma is the most common primary brain tumor among adults. Temozolomide (TMZ) is widely used as the first-line postsurgical drug for malignant glioma. However, the therapeutic efficacy of TMZ remains ineffective as inherited or acquired drug resistance is frequently observed. Estrogen receptor $\beta(E R \beta)$ has emerged as a tumor suppressor and a key regulator of signal transduction in glioma cells. However, little is known about the role of ER $\beta$ in regulating the chemotherapeutic response to TMZ. In the current study, the TMZ-resistant U138 glioma cells were treated with the novel ER $\beta$ agonist liquiritigenin (Liq). It was observed that Liq significantly enhanced ER $\beta$ expression and sensitized glioma cells to TMZ-induced proliferation inhibition. As a potential mechanism, it was noted that Liq treatment significantly inhibited the activity of the PI3K/AKT/mTOR pathway, which played a protective role against the TMZ-induced cytotoxicity. In addition, it was demonstrated that ER $\beta$ knockdown or activation of the phosphatidylinositol-4,5-bisphosphate 3-kinase (PI3K)/AKT/mammalian target of rapamycin (mTOR) pathway by insulin-like growth factor 1 both eradicated the function of Liq. These results suggest that Liq treatment enhances glioma cell susceptibility to TMZ by inhibiting the PI3K/AKT/mTOR pathway. As hyperactivation of the PI3K/AKT/mTOR pathway is frequently observed in gliomas, the combined use of ER $\beta$ agonists may become a feasible therapy option to overcome chemoresistance to TMZ.
\end{abstract}

Correspondence to: Dr Ling Jing, School of Pharmaceutical Sciences, Jilin University, 1126 Fujin Road, Changchun, Jilin 130021, P.R. China

E-mail: lingjinjlu@gmail.com

Key words: temozolomide resistance, estrogen receptor $\beta$, liquiritigenin, PI3K/AKT/mTOR

\section{Introduction}

Gliomas are the most common type of malignant primary intracranial neoplasm and are associated with high mortality and morbidity (1). Despite dramatic advances in surgical intervention and chemotherapy over the past decades, the prognosis for patients with malignant glial tumors remains poor (2). Glioblastoma multiforme is the most common and aggressive subtype of malignant glioma, with an average survival time of less than one year following diagnosis (2). Accordingly, there is an urgent requirement to develop novel and effective therapeutic strategies for this disease.

Temozolomide (TMZ) is a standard chemotherapeutic agent for gliomas following surgical resection and radiotherapy (3). Functioning as an alkylating agent, TMZ induces DNA damage via the attachment of a methyl group to guanine bases, resulting in disrupted gene expression and cell growth arrest. However, not all patients exhibit a sensitive response to TMZ, and acquired resistance to the drug following treatment is frequently observed $(4,5)$. Accordingly, an understanding of the molecular mechanisms underlying TMZ resistance is essential for the optimization of existing therapeutic strategies and the development of new ones (6).

Estrogens are steroid hormones that play a significant role in regulating the development and differentiation of the central nervous system $(7,8)$. There are two main types of cognate receptors of estrogens, ER $\alpha$ and ER $\beta$, which selectively bind to different ligands and mediate the expression of different downstream genes and signaling cascades $(7,9,10)$. $\mathrm{ER} \alpha$ has been shown to enhance the proliferation of cancer cells (10-12), while previous studies support the role of ER $\beta$ as a potential tumor suppressor $(7,10,13,14)$. Loss of ER $\beta$ expression has been repeatedly observed in high-grade glioma tumors and is associated with poor clinical outcome $(10,14,15)$. A previous study also revealed that overexpression of ER $\beta$ reduced cell proliferation in colon and breast cancer cells while knockdown of ER $\beta$ exhibited the opposite effect (13). Moreover, it has been reported that treatment with ER $\beta$-specific agonists results in proliferation arrest and/or induced cell death in a wide range of cancer 
cells $(10,11)$. Accordingly, the expression level of ER $\beta$ may be a key determinant of cellular responses to antitumor drugs, while ER $\beta$-specific agonists may serve as a potential treatment for gliomas.

Liquiritigenin (Liq) is a herb-derived, highly selective ER $\beta$ agonist (16). It activates multiple regulatory elements and downstream target genes of ER $\beta$ with high specificity, and exhibits various anti-inflammatory $(17,18)$ and antitumor effects $(19,20)$. It has been shown that Liq inhibits the production of inducible nitric oxide synthase and the release of proinflammatory cytokines in macrophages (17). There is also evidence that Liq suppresses cell proliferation in a dose- and time-dependent manner (20). Pharmacokinetic studies demonstrate that Liq exhibits strong intestinal absorption and blood-brain barrier permeability (20). These characteristics all support the therapeutic function of Liq in gliomas. However, besides its well-established role as a tumor suppressor, little is known about the function of ER $\beta$ in regulating the chemotherapeutic response of gliomas. To investigate the therapeutic advantages of the ER $\beta$ agonist, we considered whether it was possible to modulate $\operatorname{ER} \beta$ signaling using Liq and enhance cellular sensitivity to TMZ. These findings provide implications for the molecular mechanisms of TMZ resistance and have immediate clinical utility in glioma treatment.

\section{Materials and methods}

Cell lines and reagents. U138 human glioma cells were obtained from the American Type Culture Collection (Manassas, VA, USA) and cultured in Dulbecco's modified Eagle's medium supplemented with $10 \%$ fetal bovine serum (Hyclone Laboratories, Logan, UT, USA) and 1\% penicillin-streptomycin (Sigma, St. Louis, MO, USA). Liq was purchased from Biopurify Phytochemicals (Chengdu, China). AKT antibody (no. 9272), phospho-AKT antibody (no. 9271) and phospho-p70S6K antibody (no. 9205) were purchased from Cell Signaling Technology, Inc. (Danvers, MA. USA). P70S6K antibody (ab9366) was purchased from Abcam (Cambridge, UK). ER $\beta$ antibody (sc-8974), ER $\beta$ shRNA (h) lentiviral particles (sc-35325-V) and control shRNA lentiviral particles-A (sc-108080) were purchased from Santa Cruz Biotechnology, Inc. (Santa Cruz, CA, USA). All antibodies are rabbit anti-human polyclonal antibodies.

Reverse-transcription polymerase chain reaction $(P C R)$ and real-time PCR. TRIzol reagent (Invitrogen Life Technologies, Carlsbad, CA, USA) was used to extract total RNA following the manufacturer's instructions. RNA was quality checked and quantitated using Nanodrop (Thermo Fisher Scientific, Inc., Waltham, MA, USA). Total RNA $(500 \mu \mathrm{M})$ was used for cDNA synthesis with poly T primer using the Superscript III reagent kit (Invitrogen Life Technologies). Real-time PCR was performed using a 7300 real-time PCR system (Applied Biosystems, Life Technologies, Paisley, UK) using SYBR-Green PCR master mix (Bio-Rad, Hercules, CA, USA). Data are expressed as the mean \pm standard deviation of three replicates. The PCR primers used for ER $\beta$ were: 5'-GGCAGAGGACAGTAAAAGCA-3' (forward) and 5'-GGACCACACAGCAGAAAGAT-3' (reverse). For actin, they were: 5'-CCAACACAGTGCTGTCTGG-3' (forward) and 5'-TGCTGATCCACATCTGCTG-3' (reverse).

Western blotting. Cells were washed twice with ice-cold phosphate-buffered saline and lysed in protein gel buffer protein gel buffer (PGB; $60 \mathrm{mM}$ Tris- $\mathrm{HCl}, 10 \%$ SDS and $10 \%$ glycerol) with $1 \mathrm{mM}$ phenylmethanesulfonylfluoride for $20 \mathrm{~min}$ on ice. PGB buffer contains $60 \mathrm{mM}$ Tris- $\mathrm{HCl}$, $10 \%$ SDS and $10 \%$ glycerol. Cell lysates were centrifuged at $14,000 \mathrm{x}$ g for $10 \mathrm{~min}$ at $4^{\circ} \mathrm{C}$, and the supernatants were collected. Protein concentration was quantitated using Nanodrop spectrophotometer nd-1000 (Thermo Fisher Scientific, Inc.) and $10 \mu \mathrm{g}$ protein was loaded on $12 \%$ SDS-PAGE gels and transferred onto membranes (Millipore, Billerica, MA, USA). Membranes were blocked in TBST with $5 \%$ non-fat milk at room temperature for $1 \mathrm{~h}$, and incubated with primary antibodies at $4^{\circ} \mathrm{C}$ overnight. The next day, membranes were washed three times for 5 min with TBST, incubated with horseradish peroxidase-conjugated secondary antibodies for $1 \mathrm{~h}$ at room temperature and visualized using enhanced chemiluminescence (Millipore). Primary antibodies were diluted at a concentration of 1:1,000, with the exception of anti-AKT $(1: 2,000)$ and anti-ER $\beta$ (1:500).

shRNA lentiviral particle transduction. U87 cells were cultured at a density of $2 \times 10^{4}$ cells per well in 12 -well plates and cultured for $24 \mathrm{~h}$ prior to infection. The cells were approximately $50 \%$ confluent when they were infected with ER $\beta$-specific shRNA lentiviral particles or control shRNA lentiviral particles in a mixture of complete medium containing $5 \mu \mathrm{g} / \mathrm{ml}$ polybrene (Santa Cruz Biotechnology, Inc.). Following incubation for $24 \mathrm{~h}$, the culture medium was replaced with fresh complete medium and incubation was continued for $24 \mathrm{~h}$. Next, the cells were divided into three groups and transferred to new plates for subsequent assays. Knockdown efficiency was accessed using western blotting.

Cell viability assay. The effects of different agent combinations on cell viability were analyzed using the Cell Counting Kit 8 (CCK-8) cell viability assay (Dojindo, Kunamoto, Japan). Briefly, cells were suspended in 96-well plates at a density of $10^{4}$ cells per well. Following incubation for $24 \mathrm{~h}$, various agents, alone or in combination with others as indicated in the manuscript, were added to the cells. Following incubation for $72 \mathrm{~h}, 10 \mu \mathrm{l}$ CCK-8 reagent was added to each well and incubated at $37^{\circ} \mathrm{C}$ for $2 \mathrm{~h}$. Absorbance was accessed at a wavelength of 450-630 nm using a multi-mode microplate reader (BioTek Instruments, Inc., Winooski, VT, USA). Assays were performed in triplicate and cell viability was calculated as a percentage of the control. The median inhibitory concentration $\left(\mathrm{IC}_{50}\right)$ of TMZ was calculated using SPSS software 17.0 (SPSS, Inc., Chicago, IL, USA).

\section{Results}

Liq enhances expression of ER $\beta$ and sensitizes glioma cells to TMZ treatment. The TMZ-resistant U138 glioma cell line (21) was used as a model to investigate the function of Liq in modulating the cellular response to TMZ treatment. U138 cells were treated with seven different 


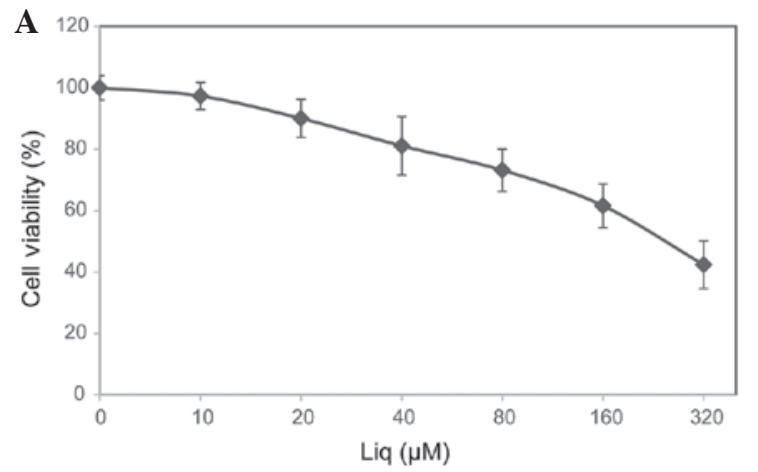

C

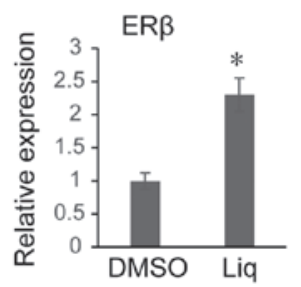

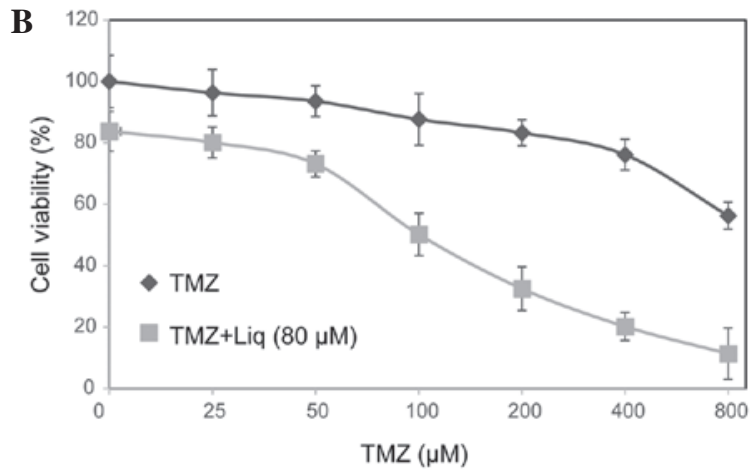

$\mathbf{E}$

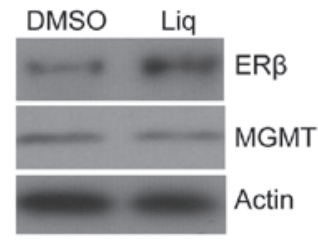

Figure 1. Liquiritigenin (Liq) treatment sensitizes glioma cells to temozolomide (TMZ)-induced proliferation inhibition. (A) Cells were treated with various doses of Liq (10-320 $\mu \mathrm{M})$ for $72 \mathrm{~h}$, and cell viability was assessed by the Cell Counting Kit 8 (CCK-8) assay. (B) Cells were treated with various doses of TMZ $(25-800 \mu \mathrm{M})$ in combination with $80 \mu \mathrm{M} \mathrm{Liq} \mathrm{for} 72 \mathrm{~h}$, and cell viability was assessed by the CCK-8 assay. All data presented are the mean \pm SD from three independent experiments. Following treatment with dimethyl sulfoxide (DMSO) or $80 \mu \mathrm{M}$ Liq for $72 \mathrm{~h}$, mRNA expression of (C) estrogen receptor $\beta$ (ER $\beta$ ) and (D) $O^{6}$-methylguanine DNA methyltransferase (MGMT) was assessed using real-time polymerase chain reaction. All data presented are the mean \pm SD from three independent experiments. (E) Western blotting of ER $\beta$ and MGMT protein expression in cells treated with DMSO or Liq.

titrations of Liq, and cell viability was determined using the CCK-8 assay after $72 \mathrm{~h}$. In agreement with a previous study (20), it was observed that Liq induced U138 cell death in a dose-dependent manner (Fig. 1A). As $80 \mu \mathrm{M} \mathrm{Liq}$ was sufficient to activate ER $\beta$ without causing severe cell death in U138 cells, this concentration of Liq was used in all subsequent assays. The effect of treatment with TMZ alone or in combination with Liq on cell viability was then investigated. Consistent with previous results (21), a clear inhibitory effect on U138 cell viability was observed only when a high dose of TMZ was used (Fig. 1B). By contrast, the combined treatment of TMZ and Liq synergistically inhibited the proliferation of U138 cells even when a low dose of TMZ was used (Fig. 1B). For cells treated with Liq and various doses of TMZ, we controlled for the influence of Liq on cell viability by normalizing viabilities of different groups to the group treated with $80 \mu \mathrm{M}$ Liq alone $(\mathrm{TMZ}=0 \mu \mathrm{M})$, and calculated a corrected $72 \mathrm{~h} \mathrm{IC}_{50}$ for $\mathrm{TMZ}$. Combined treatment with Liq significantly increased U138 susceptibility to $\mathrm{TMZ}$ (untreated $\mathrm{IC}_{50}=973.56 \mu \mathrm{M}$ vs. treated $\left.\mathrm{IC}_{50}=188.45 \mu \mathrm{M}\right)$. Since the combination of a low dose of Liq $(80 \mu \mathrm{M})$ and TMZ $(100 \mu \mathrm{M})$ could readily inhibit cell growth by $\sim 50 \%$ (Fig. 1D), this therapy option may be well tolerated with fewer side effects. Notably, using real-time PCR, it was observed that transcription activity of ER $\beta$ was notably increased following treatment with $80 \mu \mathrm{M}$ Liq for 72 h ( $\mathrm{P}=0.006$, Student's t-test; Fig. 1C). Western blotting revealed that the protein level of ER $\beta$ was also enhanced following exposure to Liq (Fig. 1E). This is in line with a previous study which demonstrated that the expression level of ER $\beta$ is self-regulated by its own ligands (22). By contrast, we noted that Liq treatment had no influence on either the transcription or protein expression of $O^{6}$-methylguanine DNA methyltransferase (MGMT) (Fig. 1D and E), which plays a significant role in repairing TMZ-induced DNA damage and regulating chemoresistance to alkylating agents $(4,5,21)$. We therefore suggest that Liq may enhance the TMZ sensitivity of U138 cells through MGMT-independent mechanisms.

Liq treatment results in inhibition of PI3K/AKT/mTOR pathway. The PI3K/AKT/mTOR pathway plays a significant role in regulating cellular processes that are critical for both normal development and tumorigenesis, including proliferation, growth, survival and mobility (23-25). There is also growing evidence to suggest that the PI3K/AKT/mTOR pathway plays a crucial role in regulating chemotherapy resistance in various tumor cells $(23,26)$. In addition, a previous study suggested that the overexpression of ER $\beta$ is associated with inhibition of the PI3K/AKT/mTOR pathway $(27,28)$. We therefore considered whether Liq modulates TMZ sensitivity by regulating the $\mathrm{PI} 3 \mathrm{~K} / \mathrm{AKT} / \mathrm{mTOR}$ pathway. After treating cells with TMZ or Liq alone and in combination, we investigated changes in the protein expression and phosphorylation of AKT and P70S6K, which are central components of this pathway, using western blotting. Notably, treatment with Liq, but not TMZ, resulted in a significant decrease in AKT and p70S6K phosphorylation (Fig. 2A). The function of the PI3K/AKT/mTOR pathway in regulating TMZ sensitivity of U138 cells was further investigated using a PI3K/mTOR dual inhibitor, XL765 (29). Notably, U138 cells treated with XL765 became more sensitive to TMZ even after controlling for the influence of XL765 (untreated $\mathrm{IC}_{50}=897.25 \mu \mathrm{M}$ vs. treated $\left.\mathrm{IC}_{50}=101.36 \mu \mathrm{M}\right)$. These results support the role of PI3K/AKT/mTOR as a key mediator of $\mathrm{TMZ}$ resistance in $\mathrm{U} 138$ cells. 
A

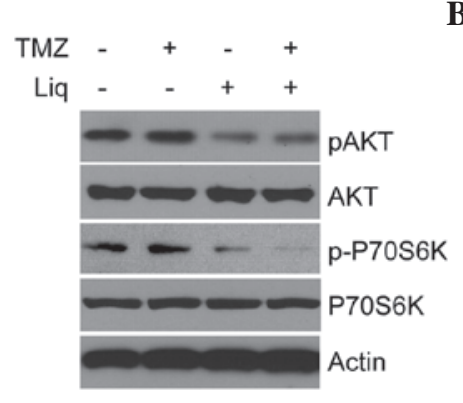

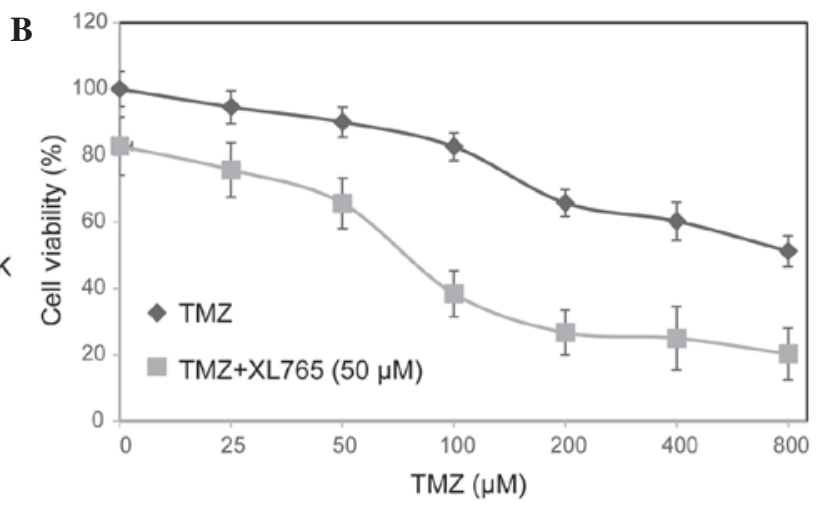

Figure 2. Liquiritigenin (Liq) treatment inhibits the activity of the phosphatidylinositol-4,5-bisphosphate 3-kinase (PI3K)/AKT/mammalian target of rapamycin (mTOR) pathway. (A) Following treatment with dimethyl sulfoxide, temozolomide (TMZ), Liq or a combination of TMZ and Liq, the phosphorylation levels of AKT and P70S6K, as well as the protein expression levels of AKT, P70S6K and actin were analyzed by western blotting. (B) Cells were treated with various doses of TMZ $(25-800 \mu \mathrm{M})$ in combination with $50 \mu \mathrm{M} \mathrm{AKT/mTOR}$ dual inhibitor XL765 for $72 \mathrm{~h}$, and cell viability was assessed by the CCK- 8 assay.

Therapeutic efficacy of Liq is ER $\beta$-dependent. Although it has been suggested that Liq targets ER $\beta$ with high specificity, it may still exhibit certain off-target activities and induce complex cellular effects. To explore whether the function of Liq in regulating TMZ resistance is ER $\beta$-dependent, we knocked down ER $\beta$ in U138 cells with lentiviral shRNA, and tested the activity of the PI3K/AKT/mTOR pathway as well as TMZ sensitivity in these cells. As shown in Fig. 3A, the expression of ER $\beta$ was markedly depressed in knockdown cells. Notably, ER $\beta$ knockdown rescued phosphorylation of AKT and P70S6K, that were otherwise missing in Liq-treated cells (Fig. 3B). Consistent with this observation, upon treatment with combined TMZ and Liq, ER $\beta$ knockdown resulted in a concomitant increase in cell proliferation compared with control cells (knockdown $\mathrm{IC}_{50}=597.35 \mu \mathrm{M}$ vs. control $\mathrm{IC}_{50}=204.28 \mu \mathrm{M}$; Fig. 3C). This was not likely to be caused by ER $\beta$ knockdown per se as knockdown cells exhibited no increase in cell viability compared with control cells when they were treated with $\mathrm{TMZ}$ alone (knockdown $\mathrm{IC}_{50}=967.35 \mu \mathrm{M}$ vs. control $\left.\mathrm{IC}_{50}=934.28 \mu \mathrm{M}\right)$. We thus conclude that the therapeutic efficacy of Liq in conferring TMZ sensitivity is dependent on $\operatorname{ER} \beta$ function.

Liq function is counterbalanced by IGF-1-induced activation of PI3K/AKT/mTOR. Through activation of ER $\beta$, Liq may regulate the activities of several signaling cascades that mediate TMZ resistance in U138 cells. This indicates that the PI3K/AKT/mTOR pathway may not necessarily be the main target of Liq/ER $\beta$ that conferred protection against TMZ-induced cell growth inhibition. To further evaluate the function of PI3K/AKT/mTOR signaling in TMZ resistance, we treated U138 cells with IGF-1 to activate this pathway and examined the cellular response to TMZ/Liq treatment. The binding of IGF-1 to its receptor causes receptor autophosphorylation and the activation of multiple signaling pathways including PI3K/AKT/mTOR signaling (30). As expected, IGF-1 treatment resulted in the superactivation of PI3K/AKT/mTOR signaling and hyperphosphorylation of AKT and p70S6K (Fig. 4A). Moreover, IGF-1 treatment largely counterbalanced the function of Liq in sensitizing U138 cells to $\mathrm{TMZ}$ (treated $\mathrm{IC}_{50}=568.35 \mu \mathrm{M}$ vs. untreated
$\mathrm{IC}_{50}=174.28 \mu \mathrm{M}$; Fig. 4B). This is consistent with the $\mathrm{PI} 3 \mathrm{~K} / \mathrm{AKT} / \mathrm{mTOR}$ pathway being significant in mediating chemoresistance (23). We thus conclude that Liq treatment sensitizes U138 cells to TMZ through inhibition of the $\mathrm{PI} 3 \mathrm{~K} / \mathrm{AKT} / \mathrm{mTOR}$ pathway.

\section{Discussion}

TMZ has emerged as a promising chemotherapy for glioblastoma; however, the clinical outcome of TMZ treatment is not always satisfactory due to the intrinsic or acquired drug resistance (2). It is well established that MGMT is one of the most significant DNA repair enzymes that targets TMZ-induced DNA damage (4). Previous studies suggest that the promoter methylation status and expression level of MGMT are associated with the susceptibility of tumor cells to TMZ $(4,5)$. Besides MGMT-dependent mechanisms, however, other mechanisms are also considered to be critical in regulating TMZ sensitivity (25). Accordingly, other pharmacological agents, through MGMT-dependent or -independent mechanisms, may readily increase the therapeutic efficacy of TMZ and increase patients' survival.

In the current study, a mechanistic link was identified between the ER $\beta$ agonist and chemoresistance to TMZ treatment. Notably, it was revealed that Liq enhanced the TMZ sensitivity of U138 cells without changing the expression level of MGMT. The results provided evidence that Liq enhanced TMZ sensitivity through inhibition of the PI3K/AKT/mTOR pathway. We also suggest that Liq function is dependent on ER $\beta$ and may be counterbalanced by the PI3K/AKT/mTOR activator IGF-1. These results provide a novel MGMT-independent mechanism for TMZ resistance and highlight the clinical use of Liq to optimize TMZ therapeutics.

$\mathrm{PI} 3 \mathrm{~K} / \mathrm{AKT} / \mathrm{mTOR}$ signaling plays a central role in regulating protein synthesis, proliferation and survival, and has been implicated in multiple drug resistance in numerous cancer types. Several components of this pathway, including epidermal growth factor receptor, Ras, PI3K and AKT, have been observed to be frequently mutated in tumor cells and are associated with the hyperactivation of the pathway (31). Independent of the MGMT function, PI3K/AKT/mTOR 
A

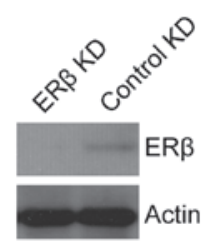

B

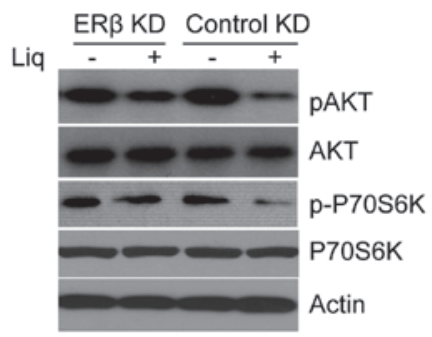

C

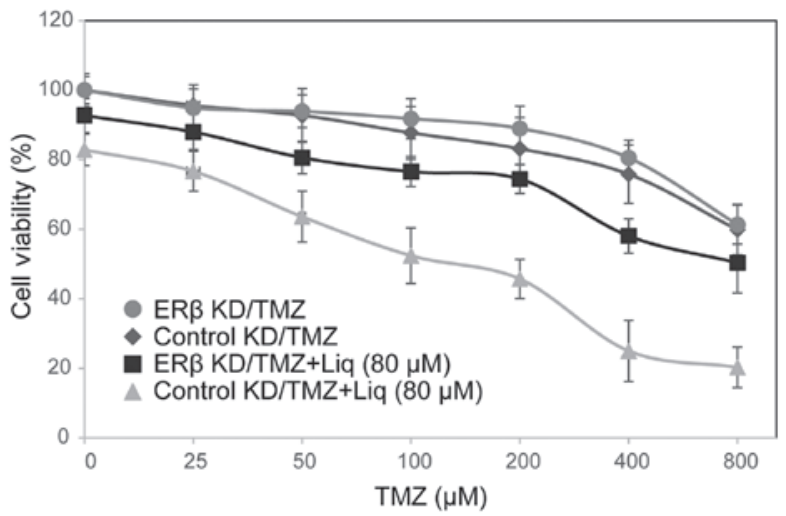

Figure 3. Liquiritigenin (Liq) function is estrogen receptor $\beta$ (ER $\beta$ )-dependent. (A) Cells were treated with ER $\beta$-specific lentiviral shRNA or control shRNA, and protein expression of ER $\beta$ was assessed using western blotting. (B) ER $\beta$ knockdown cells and control cells were treated with dimethyl sulfoxide or Liq, and the phosphorylation levels of AKT and P70S6K, as well as the protein expression levels of AKT, P70S6K and actin were analyzed by western blotting. (C) ER $\beta$ knockdown cells and control cells were treated with various doses of temozolomide (TMZ; 25-800 $\mu \mathrm{M}$ ) alone or in combination with $80 \mu \mathrm{M}$ Liq for $72 \mathrm{~h}$. Cell viability was assessed by the Cell Counting Kit 8 assay and $\mathrm{IC}_{50}$ for TMZ was calculated. KD, knockdown.

A

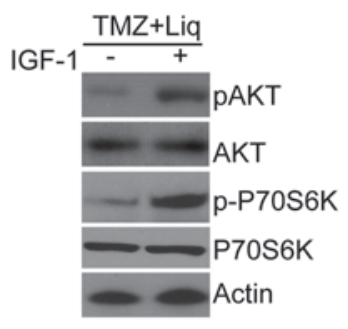

B

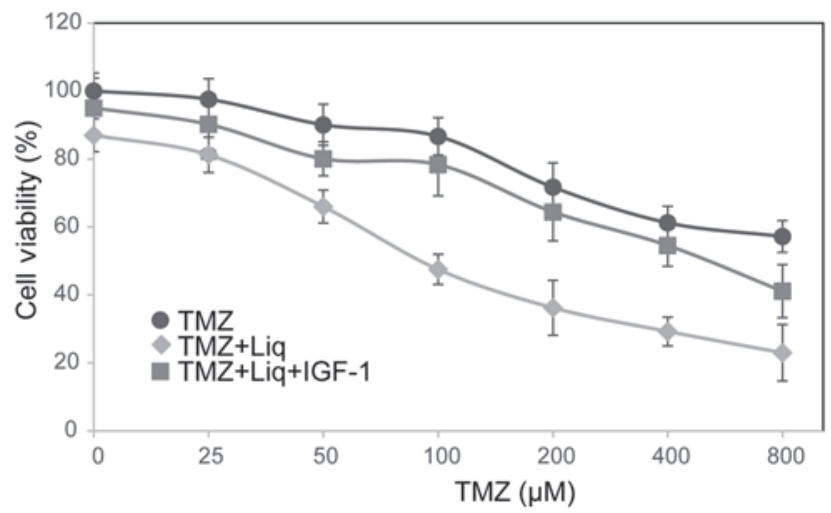

Figure 4. Activation of the phosphatidylinositol-4,5-bisphosphate 3-kinase/AKT/mammalian target of rapamycin pathway using insulin-like growth factor 1 (IGF-1)-counterbalanced liquiritigenin (Liq) function. (A) Cells were treated with temozolomide (TMZ)/Liq or TMZ/Liq/IGF-1 combination for 72 h, and phosphorylation levels of AKT and P70S6K, as well as the protein expression levels of AKT, P70S6K and actin were analyzed by western blotting. (B) Cells were treated with TMZ alone, TMZ/Liq or TMZ/Liq/IGF-1 combination for 72 h. Cell viability was assessed by the Cell Counting $\mathrm{Kit} 8$ assay and $\mathrm{IC}_{50}$ for TMZ was calculated.

signaling may facilitate the expression of a set of anti-apoptotic factors and activation of survival signals, which rid the cells of the cytotoxic effects induced by TMZ treatment $(31,32)$. For example, enhanced PI3K/AKT/mTOR activity is linked to the overexpression of c-myc, vascular endothelial growth factor and hypoxia-inducible factor- $1 \alpha(24,31)$. A previous study also revealed a mechanistic link between PI3K/AKT/mTOR hyperactivation and deregulation of homeobox A9/A10, which underlies a drug-resistant, progenitor cell phenotype in MGMT-independent pediatric glioblastoma (33). Moreover, there is also a possibility that PI3K/AKT/mTOR signaling controls cell cycle progression and mediates DNA damage repair through the non-homologous end-joining repair pathway. A previous study suggested that AKT interacts with the DNA-protein kinase catalytic subunit and induces DNA double-strand break repair (34). This provides an alternative strategy to repair TMZ-induced DNA damage and confer cell resistance to TMZ treatment. In line with these findings, it has been observed that the activated PI3K/AKT pathway promotes resistance to anti-estrogen drugs in breast cancer. Loss of phosphatase and tensin homolog (PTEN) and overexpression of $\mathrm{PI} 3 \mathrm{~K}$ are linked to cisplatin resistance in ovarian cancer cells (35). Previous studies also suggest that overexpression of PIK $3 \mathrm{CA}$ and activation of $\mathrm{PI} 3 \mathrm{~K} / \mathrm{AKT} / \mathrm{mTOR}$ signaling confer trastuzumab resistance in breast cancer (36), while constitutively active AKT is causally linked to drug resistance against tumor necrosis factor-related apoptosis-inducing ligand (37).

Accordingly, the $\mathrm{PI} 3 \mathrm{~K} / \mathrm{AKT} / \mathrm{mTOR}$ pathway is an extremely appealing therapeutic target given its significant roles in regulating multiple survival signaling and drug resistance pathways. Treatment with PI3K/AKT/mTOR inhibitors may result in growth arrest and induced cell death, while the combination of these inhibitors with other therapeutic agents often produces synergistic effects in the inhibition of tumor proliferation. For example, combination treatment of TMZ with the dual PI3K/mTOR inhibitor PI-103 resulted in a highly synergistic inhibition of cell survival in the TMZ-resistant cell line KNS42 (33), while combined treatment of dual PI3K/mTOR inhibitor XL765 enhanced TMZ-induced cytotoxicity in pituitary adenoma cells and in a mouse model (29). Our results also support the efficacy of 
targeting PI3K/AKT/mTOR using Liq as a strategy for overcoming TMZ resistance in U138 cells. We suggest that TMZ treatment in conjunction with Liq is a feasible therapy option with several benefits. Firstly, Liq was observed to be well tolerated with less neuronal toxicity in phase II and III clinical trials. Secondly, Liq possesses strong blood-brain barrier permeability and is able to reach glioma cells. Thirdly, ER $\beta$ agonist selectively targets cells expressing ER $\beta$, which have better specificity than other general PI3K/mTOR inhibitors. Finally, we suggest that in addition to its role in enhancing $\mathrm{TMZ}$ resistance, Liq may increase the expression of $\mathrm{ER} \beta$ and activate the function of this well-known tumor suppressor to inhibit tumor proliferation. We thus suggest that $\operatorname{ER} \beta$ agonists are suitable for optimizing TMZ therapies and for preventing recurrent glioma formation.

In the current study, we provide evidence that, through ER $\beta$ knockdown or PI3K/AKT/mTOR activation using IGF-1, Liq function on TMZ sensitivity is ER $\beta$-dependent and PI3K/AKT/mTOR-dependent. However, the manner in which Liq-induced ER $\beta$ activation inhibits the activity of $\mathrm{PI} 3 \mathrm{~K} / \mathrm{AKT} / \mathrm{mTOR}$ signaling requires further investigation. It has been suggested that overexpression of ER $\beta$ is linked to upregulation of PTEN and downregulation of human epidermal growth factor 2 (HER2)/HER3 (28), supporting a transcription-dependent mechanism of ER $\beta$ function. Further high-throughput studies, such as RNA-seq and proteomic studies, will help to identify downstream targets of ER $\beta$ that are involved in regulating the PI3K/AKT/mTOR pathway. Notably, given that PTEN and components of the PI3K/AKT/mTOR pathway are frequently mutated in cancer cells (38), it is conceivable that certain cells may have a weak response to ER $\beta$ signaling due to their lack of functionally intact proteins. Further attempts should be made to correlate the PTEN status and PI3K/AKT/mTOR activity with the response to the ER $\beta$ agonist in a large number of cell types and patient samples. To conclude, the results of the present study suggest that the ER $\beta$ agonist is a promising therapy for overcoming TMZ chemoresistance in human malignant glioma cells.

\section{Acknowledgements}

This study was supported in part by the National Natural Science Foundation of China (230406).

\section{References}

1. Lacroix M, Abi-Said D, Fourney DR, et al: A multivariate analysis of 416 patients with glioblastoma multiforme: prognosis, extent of resection, and survival. J Neurosurg 95: 190-198, 2001.

2. Kanu OO, Hughes B, Di C, et al: Glioblastoma multiforme oncogenomics and signaling pathways. Clin Med Oncol 3: 39-52, 2009.

3. Stupp R, Mason WP, van den Bent MJ, et al: Radiotherapy plus concomitant and adjuvant temozolomide for glioblastoma. N Engl J Med 352: 987-996, 2005.

4. Minniti G, Salvati M, Arcella A, et al: Correlation between O6-methylguanine-DNA methyltransferase and survival in elderly patients with glioblastoma treated with radiotherapy plus concomitant and adjuvant temozolomide. J Neurooncol 102: 311-316, 2011.

5. Watanabe R, Nakasu Y, Tashiro H, et al: O6-methylguanine DNA methyltransferase expression in tumor cells predicts outcome of radiotherapy plus concomitant and adjuvant temozolomide therapy in patients with primary glioblastoma. Brain Tumor Pathol 28: 127-135, 2011.
6. Yin S, Yang J, Lin B, et al: Exome sequencing identifies frequent mutation of MLL2 in non-small cell lung carcinoma from Chinese patients. Sci Rep 4: 6036, 2014.

7. Leung YK, Mak P, Hassan S and Ho SM: Estrogen receptor (ER)-beta isoforms: a key to understanding ER-beta signaling. Proc Natl Acad Sci USA 103: 13162-13167, 2006.

8. Burns KA and Korach KS: Estrogen receptors and human disease: an update. Arch Toxicol 86: 1491-1504, 2012.

9. Li LC, Yeh CC, Nojima D and Dahiya R: Cloning and characterization of human estrogen receptor beta promoter. Biochem Biophys Res Commun 275: 682-689, 2000.

10. Roepke TA, Ronnekleiv OK and Kelly MJ: Physiological consequences of membrane-initiated estrogen signaling in the brain. Front Biosci (Landmark Ed) 16: 1560-1573, 2011.

11. Liu MM, Albanese C, Anderson CM, et al: Opposing action of estrogen receptors alpha and beta on cyclin D1 gene expression. J Biol Chem 277: 24353-24360, 2002.

12. Bailey ST, Shin H, Westerling T, Liu XS and Brown M: Estrogen receptor prevents $\mathrm{p} 53$-dependent apoptosis in breast cancer. Proc Natl Acad Sci USA 109: 18060-18065, 2012.

13. Nilsson S and Gustafsson JA: Estrogen receptors: therapies targeted to receptor subtypes. Clin Pharmacol Ther 89: 44-55, 2011.

14. Paruthiyil S, Cvoro A, Zhao X, et al: Drug and cell type-specific regulation of genes with different classes of estrogen receptor beta-selective agonists. PLoS One 4: e6271, 2009.

15. Suzuki F, Akahira J, Miura I, et al: Loss of estrogen receptor beta isoform expression and its correlation with aberrant DNA methylation of the 5'-untranslated region in human epithelial ovarian carcinoma. Cancer Sci 99: 2365-2372, 2008.

16. Mersereau JE, Levy N, Staub RE, et al: Liquiritigenin is a plant-derived highly selective estrogen receptor beta agonist. Mol Cell Endocrinol 283: 49-57, 2008.

17. Kim YW, Zhao RJ, Park SJ, et al: Anti-inflammatory effects of liquiritigenin as a consequence of the inhibition of NF-kappaB-dependent iNOS and proinflammatory cytokines production. Br J Pharmacol 154: 165-173, 2008.

18. Yang EJ, Park GH and Song KS: Neuroprotective effects of liquiritigenin isolated from licorice roots on glutamate-induced apoptosis in hippocampal neuronal cells. Neurotoxicology 39: 114-123, 2013.

19. Zhou M, Higo H and Cai Y: Inhibition of hepatoma 22 tumor by Liquiritigenin. Phytother Res 24: 827-833, 2010.

20. Sareddy GR, Nair BC, Gonugunta VK, et al: Therapeutic significance of estrogen receptor beta agonists in gliomas. Mol Cancer Ther 11: 1174-1182, 2012.

21. Ryu CH, Yoon WS, Park KY, et al: Valproic acid downregulates the expression of MGMT and sensitizes temozolomide-resistant glioma cells. J Biomed Biotechnol 2012: 987495, 2012.

22. Vladusic EA, Hornby AE, Guerra-Vladusic FK, Lakins J and Lupu R: Expression and regulation of estrogen receptor beta in human breast tumors and cell lines. Oncol Rep 7: 157-167, 2000.

23. Hennessy BT, Smith DL, Ram PT, Lu Y and Mills GB: Exploiting the PI3K/AKT pathway for cancer drug discovery. Nat Rev Drug Discov 4: 988-1004, 2005.

24. Shaw RJ and Cantley LC: Ras, $\mathrm{PI}(3) \mathrm{K}$ and mTOR signalling controls tumour cell growth. Nature 441: 424-430, 2006.

25. Yin S, Wang P, Deng W, et al: Dosage compensation on the active $\mathrm{X}$ chromosome minimizes transcriptional noise of $\mathrm{X}$-linked genes in mammals. Genome Biol 10: R74, 2009.

26. Yin S, Deng W, Hu L and Kong X: The impact of nucleosome positioning on the organization of replication origins in eukaryotes. Biochem Biophys Res Commun 385: 363-368, 2009.

27. Li W, Winters A, Poteet E, et al: Involvement of estrogen receptor beta5 in the progression of glioma. Brain Res 1503: 97-107, 2013.

28. Lindberg K, Helguero LA, Omoto Y, Gustafsson JA and Haldosen LA: Estrogen receptor beta represses Akt signaling in breast cancer cells via downregulation of HER2/HER3 and upregulation of PTEN: implications for tamoxifen sensitivity. Breast Cancer Res 13: R43, 2011.

29. Dai C, Zhang B, Liu X, et al: Inhibition of PI3K/AKT/mTOR pathway enhances temozolomide-induced cytotoxicity in pituitary adenoma cell lines in vitro and xenografted pituitary adenoma in female nude mice. Endocrinology 154: 1247-1259, 2013.

30. Myers MG Jr, Grammer TC, Wang LM, et al: Insulin receptor substrate-1 mediates phosphatidylinositol 3'-kinase and p70S6k signaling during insulin, insulin-like growth factor-1, and interleukin-4 stimulation. J Biol Chem 269: 28783-28789, 1994. 
31. Jiang BH and Liu LZ: Role of mTOR in anticancer drug resistance: perspectives for improved drug treatment. Drug Resist Updat 11: 63-76, 2008.

32. Deng WJ, Nie S, Dai J, Wu JR and Zeng R: Proteome, phosphoproteome, and hydroxyproteome of liver mitochondria in diabetic rats at early pathogenic stages. Mol Cell Proteomics 9: 100-116, 2010.

33. Gaspar N, Marshall L, Perryman L, et al: MGMT-independent temozolomide resistance in pediatric glioblastoma cells associated with a PI3-kinase-mediated HOX/stem cell gene signature. Cancer Res 70: 9243-9252, 2010.

34. Toulany M, Lee KJ, Fattah KR, et al: Akt promotes post-irradiation survival of human tumor cells through initiation, progression, and termination of DNA-PKcs-dependent DNA double-strand break repair. Mol Cancer Res 10: 945-957, 2012.
35. Chock KL, Allison JM, Shimizu Y and ElShamy WM: BRCA1-IRIS overexpression promotes cisplatin resistance in ovarian cancer cells. Cancer Res 70: 8782-8791, 2010.

36. O'Brien NA, Browne BC, Chow L, et al: Activated phosphoinositide 3-kinase/AKT signaling confers resistance to trastuzumab but not lapatinib. Mol Cancer Ther 9: 1489-1502, 2010.

37. Xu J, Zhou JY, Wei WZ and Wu GS: Activation of the Akt survival pathway contributes to TRAIL resistance in cancer cells. PLoS One 5: e10226, 2010.

38. Wang SI, Puc J, Li J, et al: Somatic mutations of PTEN in glioblastoma multiforme. Cancer Res 57: 4183-4186, 1997. 